\title{
Bivariate Positive Operators in Polynomial Weighted Spaces
}

\author{
Octavian Agratini \\ Faculty of Mathematics and Computer Science, Babeş-Bolyai University, Street Kogălniceanu 1, 400084 Cluj-Napoca, Romania
}

Correspondence should be addressed to Octavian Agratini; agratini@math.ubbcluj.ro

Received 25 February 2013; Accepted 26 March 2013

Academic Editor: Sung Guen Kim

Copyright (C) 2013 Octavian Agratini. This is an open access article distributed under the Creative Commons Attribution License, which permits unrestricted use, distribution, and reproduction in any medium, provided the original work is properly cited.

\begin{abstract}
This paper aims to two-dimensional extension of some univariate positive approximation processes expressed by series. To be easier to use, we also modify this extension into finite sums. With respect to these two new classes designed, we investigate their approximation properties in polynomial weighted spaces. The rate of convergence is established, and special cases of our construction are highlighted.
\end{abstract}

\section{Introduction}

The approximation of functions by using linear positive operators is currently under research. Usually, two types of positive approximation processes are used: the discrete, respectively, continuous form. In the first case, they often are designed through a series. Since the construction of such operators requires an estimation of infinite sums, this restricts the operator usefulness from the computational point of view. In this respect, in order to approximate a function, it is interesting to consider partial sums, the number of terms considered in sum depending on the function argument. Roughly speaking, these discrete operators are truncated fading away their "tails." Thus, they become usable for generating software approximation of functions. Among the pioneers who approached this direction we mention Gróf [1] and Lehnhoff [2]. In the same direction a class of univariate linear positive operators is investigated in [3].

This work focuses on a general bivariate class of discrete positive linear operators expressed by infinite sums. This class acts in polynomial weighted spaces of continuous functions of two variables defined by $\mathbb{R}_{+} \times \mathbb{R}_{+}$, where $\mathbb{R}_{+}=[0, \infty)$. By using a certain modulus of smoothness we give theorems on the degree of approximation. Further, we replace the infinite sum by a truncated one, and we study the approximation properties of the new defined family of operators. Compared to what has been done so far, the strengths of this paper consist in using general classes of two-dimensional discrete operators, implying an arbitrary network of nodes. Finally we present some particular classes of operators that can be obtained from our family.

\section{The Operators}

Our linear and positive operators have the role to approximate functions defined on $\mathbb{R}_{+} \times \mathbb{R}_{+}$. Therefore, on this domain we define for every $(m, n) \in \mathbb{N} \times \mathbb{N}$ a net of form $\Delta_{m, n}=$ $\Delta_{1, m} \times \Delta_{2, n}$, where $\Delta_{1, m}\left(0=x_{m, 0}<x_{m, 1}<\cdots\right)$ and $\Delta_{2, n}\left(0=y_{n, 0}<y_{n, 1}<\cdots\right)$. Set $\mathbb{N}_{0}=\{0\} \cup \mathbb{N}$, and $C\left(\mathbb{R}_{+}\right)$ stands for the space of all real-valued continuous functions on $\mathbb{R}_{+}$.

Products of parametric extensions of two univariate operators are appropriate tools to approximate functions of two variables. For this reason, the starting point is given by the following one-dimensional operators:

$$
\begin{aligned}
& \left(A_{m} f\right)(x)=\sum_{i=0}^{\infty} a_{m, i}(x) f\left(x_{m, i}\right), \\
& \left(B_{n} f\right)(y)=\sum_{j=0}^{\infty} b_{n, j}(y) f\left(y_{n, j}\right),
\end{aligned}
$$

where $a_{m, i}, b_{n, j}$ are nonnegative functions belonging to $C\left(\mathbb{R}_{+}\right)$, $(i, j) \in \mathbb{N}_{0} \times \mathbb{N}_{0}$, such that the following identities

$$
\sum_{i=0}^{\infty} a_{m, i}(t)=\sum_{j=0}^{\infty} b_{n, j}(t)=1, \quad t \geq 0
$$


take place. These conditions mean that the operators $A_{m}$ and $B_{n}$ preserve the monomial $e_{0}, e_{0}(t)=1$, a property often seen at classical linear positive operators.

For each $z \in \mathbb{R}_{+}$, define the function $\varphi_{z}$ by $\varphi_{z}(t)=t-z$, $t \in \mathbb{R}_{+}$. Also, for each $r \in \mathbb{N}$, set

$$
\mathscr{M}_{r}\left(A_{m} ; x\right)=\left(A_{m} \varphi_{x}^{r}\right)(x), \quad \mathscr{M}_{r}\left(B_{n} ; y\right)=\left(B_{n} \varphi_{y}^{r}\right)(y),
$$

representing the $r$ th central moment of the specified operators.

For a simplified writing, we will use the common notation $L_{s}$, where $L_{s}=A_{s}$ for all $s \in \mathbb{N}$ or $L_{s}=B_{s}$ for all $s \in \mathbb{N}$.

For our purposes, relative to the central moments, we require additional conditions. For each $r \in \mathbb{N}, \mathscr{M}_{r}\left(L_{s} ; t\right)$ as a function of $t$ is bounded by a polynomial of degree at most $r$. Moreover, $s^{r / 2} \mathscr{M}_{r}\left(L_{s} ; \cdot\right)$ is bounded with respect to $s$. These requirements can be brought together and redrafted in the following way: for each $r \in \mathbb{N}$, a polynomial $\Gamma_{r}$ exists such that

$$
s^{r / 2} \mathscr{M}_{r}\left(L_{s} ; t\right) \leq \Gamma_{r}(t), \quad t \geq 0, \operatorname{deg}\left(\Gamma_{r}\right) \leq r .
$$

Apparently is a tough condition, but the examples that we give in the last section show that it is carried out by different classes of operators.

In what follows we specify the function spaces in which the operators act.

For univariate operators $A_{s}, B_{s}$ we consider the space $C_{p}\left(\mathbb{R}_{+}\right), p \in \mathbb{N}_{0}$ fixed, consisting of all real-valued functions $f$ continuous on $\mathbb{R}_{+}$such that $w_{p} f$ is uniformly continuous and bounded on $\mathbb{R}_{+}$, where the weight $w_{p}$ is defined as follows:

$$
w_{0}(t)=1, \quad w_{p}(t)=\left(1+t^{p}\right)^{-1} \quad \text { for } p \in \mathbb{N} .
$$

The space is endowed with the norm $\|\cdot\|_{p},\|f\|_{p}=$ $\sup _{x \geq 0} w_{p}(x)|f(x)|$.

For bivariate operators we consider the space

$C_{p, q}\left(\mathbb{R}_{+}^{2}\right)=\left\{f \in C\left(\mathbb{R}_{+}^{2}\right): w_{p, q} f\right.$ is uniformly continuous and bounded on $\left.\mathbb{R}_{+}^{2}\right\}$

associated to the weighted function $w_{p, q}(x, y)=w_{p}(x) w_{q}(y)$, $(p, q) \in \mathbb{N}_{0} \times \mathbb{N}_{0}$. The norm of this space is denoted by $\|\cdot\|_{p, q}$ and is defined by

$$
\|f\|_{p, q}=\sup _{(x, y) \in \mathbb{R}_{+}^{2}} w_{p, q}(x, y)|f(x, y)| .
$$

These spaces are ordered with respect to inclusion as follows: if $(p, q) \leq\left(p^{\prime}, q^{\prime}\right)$, then $C_{p, q}\left(\mathbb{R}_{+}^{2}\right) \subset C_{p^{\prime}, q^{\prime}}\left(\mathbb{R}_{+}^{2}\right)$. Moreover, $\|f\|_{p^{\prime}, q^{\prime}} \leq\|f\|_{p, q}$ for any $f \in C_{p, q}\left(\mathbb{R}_{+}^{2}\right)$. Examining the weight $w_{p, q}$, it is easy to verify the inequality

$$
\left|\int_{x}^{t} \frac{d u}{w_{p, q}(u, z)}\right| \leq|t-x|\left(\frac{1}{w_{p, q}(x, z)}+\frac{1}{w_{p, q}(t, z)}\right),
$$

for any $x \geq 0$ and $t \geq 0$.
Indeed, based on the first mean value theorem for integration, between $x$ and $t$, a point $\xi_{x, t}$ exists such that

$$
\begin{aligned}
\left|\int_{x}^{t} \frac{d u}{w_{p, q}(u, z)}\right| & =|t-x|\left(1+\xi_{x, t}^{p}\right)\left(1+z^{q}\right) \\
& \leq|t-x|\left(1+x^{p}+1+t^{p}\right)\left(1+z^{q}\right) \\
& =|t-x|\left(\frac{1}{w_{p, q}(x, z)}+\frac{1}{w_{p, q}(t, z)}\right) .
\end{aligned}
$$

Starting from (1), for each $(m, n) \in \mathbb{N} \times \mathbb{N}$ we introduce a linear positive operator in polynomial weighted space $C_{p, q}\left(\mathbb{R}_{+}^{2}\right)$ as follows:

$$
\begin{array}{r}
\left(L_{m, n} f\right)(x, y)=\sum_{i=0}^{\infty} \sum_{j=0}^{\infty} a_{m, i}(x) b_{n, j}(y) f\left(x_{m, i}, y_{n, j}\right), \\
(x, y) \in \mathbb{R}_{+}^{2} .
\end{array}
$$

If the function $f \in C_{p, q}\left(\mathbb{R}_{+}^{2}\right)$ can be decomposed in the following manner $f(x, y)=f_{1}(x) f_{2}(y),(x, y) \in \mathbb{R}_{+}^{2}$, then one has

$$
\left(L_{m, n} f\right)(x, y)=\left(A_{m} f_{1}\right)(x)\left(B_{n} f_{2}\right)(y)
$$

For example, we get $L_{m, n} w_{p, q}=\left(A_{m} w_{p}\right)\left(B_{n} w_{q}\right)$.

In order to present the rate of convergence for our bivariate operators, we use a modulus of smoothness associated to any function $f$ belonging to $C_{p, q}\left(\mathbb{R}_{+}^{2}\right)$. It is given by the formula

$$
\omega_{f}(h, \delta)=\sup _{\substack{0 \leq u \leq h \\ 0 \leq v \leq \delta}}\left\|\Delta_{u, v} f\right\|_{p, q}, \quad(h, \delta) \in \mathbb{R}_{+}^{2},
$$

where

$$
\Delta_{u, v} f(x, y)=f(x+u, y+v)-f(x, y)
$$

for $(x, y)$ and $(u, v)$ belonging to $C_{p, q}\left(\mathbb{R}_{+}^{2}\right)$. Alternative notation is $\omega(f ; x, y)$. More information about moduli of smoothness can be found in the monograph [4].

Further, we indicate a truncated variant of operators defined at $(10)$. Let $\left(u_{s}\right)_{s \geq 1},\left(v_{s}\right)_{s \geq 1}$ be strictly increasing sequences of positive numbers such that

$$
\lim _{s \rightarrow \infty} \sqrt{s} u_{s}=\lim _{s \rightarrow \infty} \sqrt{s} v_{s}=\infty
$$

Taking in view the net $\Delta_{1, m}$, we partitioned the set $\mathbb{N}_{0}$ into two parts

$$
\begin{gathered}
I\left(x, u_{m}\right)=\left\{i \in \mathbb{N}_{0}: x_{m, i} \leq x+u_{m}\right\}, \\
\bar{I}\left(x, u_{m}\right)=\mathbb{N}_{0} \backslash I\left(x, u_{m}\right) .
\end{gathered}
$$

Similarly, via the network $\Delta_{2, n}$, we introduce $J\left(y, v_{n}\right)$ and $\bar{J}\left(y, v_{n}\right)$. 
For each $(m, n) \in \mathbb{N} \times \mathbb{N}$ and any $f \in C_{p, q}\left(\mathbb{R}_{+}^{2}\right)$ we define the linear positive operators

$$
\begin{array}{r}
\left(L_{m, n}^{\left\langle u_{m}, v_{n}\right\rangle} f\right)(x, y) \\
=\sum_{i \in I\left(x, u_{m}\right)} \sum_{j \in J\left(y, v_{n}\right)} a_{m, i}(x) b_{n, j}(y) f\left(x_{m, i}, y_{n, j}\right), \\
(x, y) \in \mathbb{R}_{+}^{2} .
\end{array}
$$

\section{Auxiliary Results}

Throughout the paper, by $c(\cdot)$ we denote different real constants, in the brackets specifying the parameter(s) that the indicated constant depends.

At first we collect some useful results relative to the onedimensional operators $L_{s}$ where $L_{s}=A_{s}(s \in \mathbb{N})$ or $L_{s}=$ $B_{s}(s \in \mathbb{N})$.

Lemma 1. Let $p \in \mathbb{N}_{0}$, and let the weight $w_{p}$ be given by (5). The operator $L_{s}$ satisfies

$$
\begin{gathered}
\text { (i) } w_{p}(t) L_{s}\left(\frac{1}{w_{p}} ; t\right) \leq c(p), \\
\text { (ii) } w_{p}(t)\left|\left(L_{s} f\right)(t)\right| \leq c(p)\|f\|_{p}, \\
\left\|L_{s} f\right\|_{p} \leq c(p)\|f\|_{p},
\end{gathered}
$$

where $t \geq 0$ and $s \in \mathbb{N}$.

Proof. Let $p \in \mathbb{N}_{0}$ and $s \in \mathbb{N}$ be fixed.

(i) By using (5), (2), (3), and (4) we can write

$$
\begin{aligned}
w_{p}(t) & L_{s}\left(\frac{1}{w_{p}} ; t\right) \\
& =w_{p}(t) L_{s}\left(1+((x-t)+t)^{p} ; t\right) \\
& =w_{p}(t)\left(1+t^{p}+\sum_{k=1}^{p}\left(\begin{array}{l}
p \\
k
\end{array}\right) L_{s}\left((x-t)^{k} t^{p-k} ; t\right)\right) \\
& =1+\sum_{k=1}^{p}\left(\begin{array}{l}
p \\
k
\end{array}\right) \frac{t^{p-k} \mathscr{M}_{k}\left(L_{s} ; t\right)}{1+t^{p}} \\
& \leq 1+\sum_{k=1}^{p}\left(\begin{array}{l}
p \\
k
\end{array}\right) \frac{t^{p-k} \Gamma_{k}(t)}{1+t^{p}} .
\end{aligned}
$$

Since $\operatorname{deg}\left(t^{p-k} \Gamma_{k}(t)\right) \leq p$, the previous expression is bounded with respect to $t \in \mathbb{R}_{+}$, and inequality (17) follows.

(ii) To be more explicit we consider $L_{s}=A_{s}$

$$
\begin{aligned}
w_{p}(t)\left|\left(A_{s} f\right)(t)\right| & \leq w_{p}(t) \sum_{k=0}^{\infty} a_{s, k}(t)\left(w_{p}|f|\right)\left(x_{s, k}\right) \frac{1}{w_{p}\left(x_{s, k}\right)} \\
& \leq\|f\|_{p} w_{p}(t) A_{s}\left(\frac{1}{w_{p}} ; t\right) .
\end{aligned}
$$

By using (17) we obtain the first inequality of the relation (18). Further, applying sup $\operatorname{sun}_{t \geq 0}$, the second inequality is proved.

Lemma 2. Let $p \in \mathbb{N}_{0}$, and let the weight $w_{p}$ be given by (5). For any $s \in \mathbb{N}$ the operator $L_{s}$ satisfies

$$
\text { (i) } w_{p}(t) L_{s}\left(\frac{\varphi_{t}^{2}}{w_{p}} ; t\right) \leq \frac{\widetilde{\Gamma}(t)}{s}, \quad t \geq 0
$$

$$
\text { (ii) } w_{p}(t) L_{s}\left(\frac{\left|\varphi_{t}\right|}{w_{p}} ; t\right) \leq c(p) \frac{\sqrt{\widetilde{\Gamma}(t)}}{\sqrt{s}}, \quad t \geq 0 \text {, }
$$

where $\varphi_{t}(x)=x-t$ and $\widetilde{\Gamma}=\Gamma_{2}+\sum_{k=0}^{p}\left(\begin{array}{l}p \\ k\end{array}\right) \Gamma_{k+2}$. The polynomials $\Gamma_{v}, v \geq 2$, are introduced by (4).

Proof. Let $p \in \mathbb{N}_{0}$ and $s \in \mathbb{N}$ be fixed.

(i) Using the identity

$$
1+t^{p}=1+\sum_{k=0}^{p}\left(\begin{array}{l}
p \\
k
\end{array}\right)(x-t)^{k} t^{p-k}
$$

we can write the following:

$$
\begin{aligned}
w_{p}(t) L_{s}\left(\frac{\varphi_{t}^{2}}{w_{p}} ; t\right)= & \frac{1}{1+t^{p}} L_{s}\left(\varphi_{t}^{2} ; t\right) \\
& +\sum_{k=0}^{p} \frac{t^{p-k}}{1+t^{p}}\left(\begin{array}{l}
p \\
k
\end{array}\right) L_{s}\left(\varphi_{t}^{k+2} ; t\right) \\
\leq & \mathscr{M}_{2}\left(L_{s} ; t\right)+\sum_{k=0}^{p}\left(\begin{array}{l}
p \\
k
\end{array}\right) \mathscr{M}_{k+2}\left(L_{s} ; t\right) \\
\leq & \frac{\Gamma_{2}(t)}{s}+\sum_{k=0}^{p}\left(\begin{array}{l}
p \\
k
\end{array}\right) \frac{\Gamma_{k+2}(t)}{s^{1+k / 2}} .
\end{aligned}
$$

During the previous relations we used notation (3) and hypothesis (4). Considering the significance of $\widetilde{\Gamma}$, relation (21) follows.

(ii) Taking into consideration relation (1), we apply the Cauchy-Schwarz inequality, and this allows us to write

$$
\begin{aligned}
w_{p}(t) & L_{s}\left(\frac{\left|\varphi_{t}\right|}{w_{p}} ; t\right) \\
& \leq\left(w_{p}(t) L_{s}\left(\frac{1}{w_{p}} ; t\right)\right)^{1 / 2}\left(w_{p}(t) L_{s}\left(\frac{\varphi_{t}^{2}}{w_{p}} ; t\right)\right)^{1 / 2} .
\end{aligned}
$$

Relations (17) and (21) imply (22). 
We mention that with the help of the same CauchySchwarz inequality, relations (2) and (4) lead us to the following inequality:

$$
L_{s}\left(\left|\varphi_{t}\right|^{r} ; t\right) \leq \sqrt{\frac{\Gamma_{2 r}(t)}{s^{r}}}, \quad t \geq 0
$$

Lemma 1 leads to the following result.

Lemma 3. Let $(p, q) \in \mathbb{N}_{0} \times \mathbb{N}_{0}$. For any $(m, n) \in \mathbb{N} \times \mathbb{N}$, the operator $L_{m, n}$ given by (10) verifies

$$
\text { (i) }\left\|L_{m, n}\left(\frac{1}{w_{p, q}} ; \cdot\right)\right\|_{p, q} \leq c(p, q) \text {, }
$$

(ii) $\left\|L_{m, n} f\right\|_{p, q} \leq c(p, q)\|f\|_{p, q}, \quad f \in C_{p, q}\left(\mathbb{R}_{+}^{2}\right)$.

Proof. The first statement follows immediately from the definition of the weight $w_{p, q}$ and relations (11), (17).

Regarding the second statement, based on (10), we get

$$
\begin{aligned}
& w_{p, q}(x, y)\left|\left(L_{m, n} f\right)(x, y)\right| \\
& \quad \leq w_{p, q}(x, y)\|f\|_{p, q} L_{m, n}\left(\frac{1}{w_{p, q}} ; x, y\right) .
\end{aligned}
$$

Applying $\sup _{(x, y) \in \mathbb{R}_{+}^{2}}$ and taking into account (27), one obtains (28).

In our investigation we appeal to the Steklov function. This can be used to approximate continuous functions by smoother functions. The Steklov function associated with $f \in$ $C\left(\mathbb{R}_{+}^{2}\right)$ is given as follows:

$$
f_{h, \delta}(x, y)=\frac{1}{h \delta} \int_{0}^{h} d u \int_{0}^{\delta} f(x+u, y+v) d v, \quad(x, y) \in \mathbb{R}_{+}^{2},
$$

where $h>0$ and $\delta>0$. By using (13) we deduce

$$
f_{h, \delta}(x, y)-f(x, y)=\frac{1}{h \delta} \int_{0}^{h} d u \int_{0}^{\delta} \Delta_{u, v} f(x, y) d v .
$$

In the next lemma we have gathered some known properties of Steklov function $f_{h, \delta}$, where $f \in C_{p, q}\left(\mathbb{R}_{+}^{2}\right)$. These properties establish connections between $f_{h, \delta}$ and the modulus $\omega_{f}$ indicated at (12). For the sake of completeness we present the proofs of these inequalities.

Lemma 4. Let $f$ belong to $C_{p, q}\left(\mathbb{R}_{+}^{2}\right)$, and let $f_{h, \delta}$ be defined by (30). The following relations take place:

$$
\text { (i) }\left\|f_{h, \delta}-f\right\|_{p, q} \leq \omega_{f}(h, \delta) \text {, }
$$

$$
\text { (ii) }\left\|\frac{\partial}{\partial x} f_{h, \delta}\right\|_{p, q} \leq \frac{2}{h} \omega_{f}(h, \delta), \quad\left\|\frac{\partial}{\partial y} f_{h, \delta}\right\| \leq \frac{2}{\delta} \omega_{f}(h, \delta),
$$

where $h>0, \delta>0$, and $\omega_{f}$ is defined by (12).
Proof. Let $h>0$ and $\delta>0$ be arbitrarily fixed.

(i) For $u \in[0, h]$ and $v \in[0, \delta]$ we deduce

$$
\left|\Delta_{u, v} f(x, y)\right| \leq \sup _{\substack{0 \leq \tau_{1} \leq h \\ 0 \leq \tau_{2} \leq \delta}}\left|\Delta_{\tau_{1}, \tau_{2}} f(x, y)\right|
$$

On the other hand,

$$
\sup _{(x, y) \in \mathbb{R}_{+}^{2}} w_{p, q}(x, y)\left|\Delta_{\tau_{1}, \tau_{2}} f(x, y)\right|=\left\|\Delta_{\tau_{1}, \tau_{2}} f\right\|_{p, q} .
$$

Keeping in mind (31), we consequently obtain

$$
\left\|f_{h, \delta}-f\right\|_{p, q} \leq \frac{1}{h \delta} \sup _{\substack{0 \leq \tau_{1} \leq h \\ 0 \leq \tau_{2} \leq \delta}}\left\|\Delta_{\tau_{1}, \tau_{2}} f\right\|_{p, q} \int_{0}^{h} d u \int_{0}^{\delta} d v=\omega_{f}(h, \delta)
$$

and (32) is completed.

(ii) We justify only the first inequality, and the second inequality can be proven in the same manner.

Occurs

$$
\begin{aligned}
\frac{\partial}{\partial x}\left(\int_{0}^{h} f(x+u, y+v) d u\right)= & f(x+h, y+v) \\
& -f(x, y+v) \\
= & \Delta_{h, v} f(x, y)-\Delta_{0, v} f(x, y)
\end{aligned}
$$

(see (13)). Further, we can write

$$
\begin{aligned}
& \left\|\frac{\partial}{\partial x} f_{h, \delta}\right\|_{p, q} \\
& =\frac{1}{h \delta} \sup _{\substack{x \geq 0 \\
y \geq 0}} w_{p, q}(x, y)\left|\int_{0}^{\delta}\left(\Delta_{h, v} f(x, y)-\Delta_{0, v} f(x, y)\right) d v\right| \\
& \leq \frac{1}{h \delta} \sup _{\substack{x \geq 0 \\
y \geq 0}} w_{p, q}(x, y) \int_{0}^{\delta}\left|\Delta_{h, v} f(x, y)\right| d v \\
& \quad+\frac{1}{h \delta} \sup _{\substack{x \geq 0 \\
y \geq 0}} w_{p, q}(x, y) \int_{0}^{\delta}\left|\Delta_{0, v} f(x, y)\right| d v \\
& :=I_{1}+I_{2} .
\end{aligned}
$$

Since $0 \leq v \leq \delta$, it is clear that $\left|\Delta_{h, v} f(x, y)\right| \leq$ $\sup _{\substack{0 \leq \tau_{1} \leq h \\ 0 \leq \tau_{2} \leq \delta}}\left|\Delta_{\tau_{1}, \tau_{2}} f(x, y)\right|$, and we obtain

$$
\begin{aligned}
I_{1} & \leq \frac{1}{h \delta} \sup _{\substack{0 \leq \tau_{1} \leq h \\
0 \leq \tau_{2} \leq \delta}} \int_{0}^{\delta} \sup _{\substack{x \geq 0 \\
y \geq 0}} w_{p, q}(x, y)\left|\Delta_{\tau_{1}, \tau_{2}} f(x, y)\right| d v \\
& \leq \frac{1}{h} \sup _{\substack{0 \leq \tau_{1} \leq h \\
0 \leq \tau_{2} \leq \delta}}\left\|\Delta_{\tau_{1}, \tau_{2}}\right\|_{p, q}=\frac{1}{h} \omega_{f}(h, \delta) .
\end{aligned}
$$

In the same manner we show $I_{2} \leq(1 / h) \omega_{f}(h, \delta)$. Returning at (38), the proof is ended. 
In the following we denote by $C_{p, q}^{1}\left(\mathbb{R}_{+}^{2}\right)$ the space of all functions $g: \mathbb{R}_{+}^{2} \rightarrow \mathbb{R}$ having the first order partial derivatives such that the functions $\partial g / \partial x, \partial g / \partial y$, and $g$ belong to $C_{p, q}\left(\mathbb{R}_{+}^{2}\right)$.

Lemma 5. Let $(p, q) \in \mathbb{N}_{0} \times \mathbb{N}_{0}$. If $g \in C_{p, q}^{1}\left(\mathbb{R}_{+}^{2}\right)$, then for any $(m, n) \in \mathbb{N} \times \mathbb{N}$ the operator $L_{m, n}$ given by $(10)$ verifies

$$
\begin{array}{r}
w_{p, q}(x, y)\left|\left(L_{m, n} g\right)(x, y)-g(x, y)\right| \\
\leq c(p, q)\left(\left\|\frac{\partial g}{\partial x}\right\|_{p, q} \frac{\Phi(x)}{\sqrt{m}}+\left\|\frac{\partial g}{\partial y}\right\|_{p, q} \frac{\Phi(y)}{\sqrt{n}}\right), \\
(x, y) \in \mathbb{R}_{+}^{2},
\end{array}
$$

where

$$
\Phi(t)=\sqrt{\Gamma_{2}(t)}+\sqrt{\Gamma_{2}(t)+\sum_{k=0}^{p}\left(\begin{array}{l}
p \\
k
\end{array}\right) \Gamma_{k+2}(t)},
$$

the polynomials $\Gamma_{v}, v=\overline{2, p+2}$, being indicated at (4).

Proof. Let $(x, y) \in \mathbb{R}_{+}^{2}$ and $(m, n) \in \mathbb{N}^{2}$ be arbitrarily fixed. Since $g \in C_{p, q}^{1}\left(\mathbb{R}_{+}^{2}\right)$, for any $(t, z) \in \mathbb{R}_{+}^{2}$ we can write

$$
g(t, z)-g(x, y)=\int_{x}^{t} \frac{\partial}{\partial u} g(u, z) d u+\int_{y}^{z} \frac{\partial}{\partial v} g(x, v) d v .
$$

Since $L_{m, n}$ is linear monotone and reproduces the constants, from the previous identity we obtain

$$
\begin{aligned}
\left|\left(L_{m, n} g\right)(x, y)-g(x, y)\right| \\
=\mid L_{m, n}\left(\int_{x}^{t} \frac{\partial}{\partial u} g(u, z) d u ; x, y\right) \\
\quad+L_{m, n}\left(\int_{y}^{z} \frac{\partial}{\partial v} g(x, v) d v ; x, y\right) \mid \\
\leq L_{m, n}\left(\left|\int_{x}^{t} \frac{\partial}{\partial u} g(u, z) d u\right| ; x, y\right) \\
\quad+L_{m, n}\left(\left|\int_{y}^{z} \frac{\partial}{\partial v} g(x, v) d v\right| ; x, y\right) \\
:=J_{1}+J_{2} .
\end{aligned}
$$

Further, one has

$$
\begin{aligned}
& \left|\int_{x}^{t} \frac{\partial}{\partial u} g(u, z) d u\right| \\
& \quad \leq\left|\int_{x}^{t} w_{p, q}(u, z)\right| \frac{\partial}{\partial u} g(u, z)\left|\frac{d u}{w_{p, q}(u, z)}\right| \\
& \quad \leq\left\|\frac{\partial g}{\partial x}\right\|_{p, q}\left|\int_{x}^{t} \frac{d u}{w_{p, q}(u, z)}\right| \\
& \quad \leq\left\|\frac{\partial g}{\partial x}\right\|_{p, q}\left(\frac{1}{w_{p, q}(x, z)}+\frac{1}{w_{p, q}(t, z)}\right)|t-x|,
\end{aligned}
$$

see (8). Applying $L_{m, n}$ and by using successively (11), (26), (17), and (22) we have

$$
\begin{aligned}
& J_{1} \leq\left\|\frac{\partial g}{\partial x}\right\|_{p, q}( L_{m, n}\left(\frac{|t-x|}{w_{p, q}(x, z)} ; x, y\right) \\
&\left.\quad+L_{m, n}\left(\frac{|t-x|}{w_{p, q}(t, z)} ; x, y\right)\right) \\
&=\left\|\frac{\partial g}{\partial x}\right\|_{p, q}( \frac{1}{w_{p}(x)} A_{m}\left(\left|\varphi_{x}\right| ; x\right) B_{n}\left(\frac{1}{w_{q}} ; y\right) \\
&\left.\quad+A_{m}\left(\frac{\left|\varphi_{x}\right|}{w_{p}} ; x\right) B_{n}\left(\frac{1}{w_{q}} ; y\right)\right) \\
& \leq\left\|\frac{\partial g}{\partial x}\right\|_{p, q}\left(\frac{1}{w_{p}(x)} \sqrt{\left.\frac{\Gamma_{2}(x)}{m}+\frac{c(p)}{w_{p}(x)} \sqrt{\frac{\widetilde{\Gamma}(x)}{m}}\right) \frac{c(q)}{w_{q}(y)}}\right. \\
& \leq \frac{c(p, q)}{w_{p, q}(x, y)}\left\|\frac{\partial g}{\partial x}\right\|_{p, q} \frac{\sqrt{\Gamma_{2}(x)}+\sqrt{\widetilde{\Gamma}(x)}}{\sqrt{m}},
\end{aligned}
$$

where $c(p, q)$ is a suitable constant. Following the same pathway, we find

$$
J_{1} \leq \frac{c(p, q)}{w_{p, q}(x, y)}\left\|\frac{\partial g}{\partial y}\right\|_{p, q} \frac{\sqrt{\Gamma_{2}(y)}+\sqrt{\widetilde{\Gamma}(y)}}{\sqrt{n}} .
$$

Considering the increases established for $J_{1}, J_{2}$ and returning to the relation (43), the inequality (40) is completely proven.

\section{Main Results}

The rate of convergence for $L_{m, n}$ operator will be read as follows.

Theorem 6. Let $(p, q) \in \mathbb{N}_{0} \times \mathbb{N}_{0}$. For any $(m, n) \in \mathbb{N} \times \mathbb{N}$, the operator $L_{m, n}$ given by (10) satisfies

$$
\begin{array}{r}
w_{p, q}(x, y)\left|\left(L_{m, n} f\right)(x, y)-f(x, y)\right| \\
\leq c(p, q) \omega_{f}\left(\frac{\Phi(x)}{\sqrt{m}}, \frac{\Phi(y)}{\sqrt{n}}\right),
\end{array}
$$

$(x, y) \in \mathbb{R}_{+}^{2}$, where $\Phi$ is given by $(41)$ and $c(p, q)$ is a suitable constant.

Proof. Setting

$$
\begin{gathered}
T_{1}:=w_{p, q}(x, y)\left|L_{m, n}\left(f-f_{h, \delta} ; x, y\right)\right|, \\
T_{2}:=w_{p, q}(x, y)\left|\left(L_{m, n} f_{h, \delta}\right)(x, y)-f_{h, \delta}(x, y)\right|, \\
T_{3}:=w_{p, q}(x, y)\left|f_{h, \delta}(x, y)-f(x, y)\right|,
\end{gathered}
$$

we can write

$$
w_{p, q}(x, y)\left|\left(L_{m, n} f\right)(x, y)-f(x, y)\right| \leq T_{1}+T_{2}+T_{3} .
$$


We establish upper bounds for these three quantities. Relations (28) and (32) imply that

$$
\begin{aligned}
T_{1} & \leq\left\|L_{m, n}\left(f-f_{h, \delta} ; x, y\right)\right\|_{p, q} \leq c(p, q)\left\|f-f_{h, \delta}\right\|_{p, q} \\
& \leq c(p, q) \omega_{f}(h, \delta) .
\end{aligned}
$$

For $T_{2}$ we use Lemma 5 choosing $g=f_{h, \delta} \in C_{p, q}^{1}\left(\mathbb{R}_{+}^{2}\right)$; see definition (31).

One has

$$
\begin{aligned}
T_{2} & \leq c(p, q)\left(\left\|\frac{\partial f_{h, \delta}}{\partial x}\right\|_{p, q} \frac{\Phi(x)}{\sqrt{m}}+\left\|\frac{\partial f_{h, \delta}}{\partial y}\right\|_{p, q} \frac{\Phi(y)}{\sqrt{n}}\right) \\
& \leq c(p, q)\left(\frac{2 \Phi(x)}{h \sqrt{m}}+\frac{2 \Phi(y)}{\delta \sqrt{n}}\right) \omega_{f}(h, \delta)
\end{aligned}
$$

(see also (33)). Finally, inequality (32) implies

$$
T_{3} \leq\left\|f_{h, \delta}-f\right\|_{p, q} \leq \omega_{f}(h, \delta) .
$$

Setting $h=\Phi(x) / \sqrt{m}, \delta=\Phi(y) / \sqrt{n}$ and coming back to (49), we can affirm that a certain constant $c(p, q)$ exists such that (47) holds.

Knowing that the modulus $\omega_{f}$ enjoys the property $\lim _{(h, \delta) \rightarrow\left(0^{+}, 0^{+}\right)} \omega_{f}(h, \delta)=0$, from Theorem 6 we deduce the following result.

Theorem 7. Let $(p, q) \in \mathbb{N}_{0} \times \mathbb{N}_{0}$, and let the operators $L_{m, n}$, $(m, n) \in \mathbb{N} \times \mathbb{N}$, be defined by $(10)$.

For any $(x, y) \in \mathbb{R}_{+}^{2}$ the pointwise convergence takes place

$$
\lim _{m, n \rightarrow \infty}\left(L_{m, n} f\right)(x, y)=f(x, y), \quad f \in C_{p, q}\left(\mathbb{R}_{+}^{2}\right) .
$$

If $K_{1}, K_{2}$ are compact intervals included in $\mathbb{R}_{+}$, then (53) holds uniformly on the domain $K_{1} \times K_{2}$.

Theorem 8. Let $(p, q) \in \mathbb{N}_{0} \times \mathbb{N}_{0}$, and let the operators $L_{m, n}^{\left\langle u_{m}, v_{n}\right\rangle}$, $(m, n) \in \mathbb{N} \times \mathbb{N}$, be defined by (16). For any $(x, y) \in \mathbb{R}_{+}^{2}$ the pointwise convergence takes place

$$
\lim _{m, n \rightarrow \infty}\left(L_{m, n}^{\left\langle u_{m}, v_{n}\right\rangle} f\right)(x, y)=f(x, y), \quad f \in C_{p, q}\left(\mathbb{R}_{+}^{2}\right) .
$$

If $K_{1}, K_{2}$ are compact intervals included in $\mathbb{R}_{+}$, then (54) holds uniformly on the domain $K_{1} \times K_{2}$.

Proof. Let $(x, y) \in \mathbb{R}_{+}^{2}$ be arbitrarily fixed. Taking in view the partitions of $\mathbb{N}_{0}$ (see (15)), we use the following decomposition:

$$
\mathbb{N}_{0} \times \mathbb{N}_{0}=(I \times J) \cup(\bar{I} \times \bar{J}) \cup(\bar{I} \times J) \cup(I \times \bar{J}) .
$$

\section{Setting}

$$
\begin{aligned}
& \left({ }_{1} R_{m, n}^{\left\langle u_{m}, v_{n}\right\rangle} f\right)(x, y) \\
& =\sum_{i \in \bar{I}\left(x, u_{m}\right)} \sum_{j \in \bar{J}\left(y, v_{n}\right)} a_{m, i}(x) b_{n, j}(y) f\left(x_{m, i}, y_{n, j}\right), \\
& \left({ }_{2} R_{m, n}^{\left\langle u_{m}, v_{n}\right\rangle} f\right)(x, y) \\
& =\sum_{i \in \bar{I}\left(x, u_{m}\right)} \sum_{j \in J\left(y, v_{n}\right)} a_{m, i}(x) b_{n, j}(y) f\left(x_{m, i}, y_{n, j}\right), \\
& \left({ }_{3} R_{m, n}^{\left\langle u_{m}, v_{n}\right\rangle} f\right)(x, y) \\
& =\sum_{i \in I\left(x, u_{m}\right)} \sum_{j \in \bar{J}\left(y, v_{n}\right)} a_{m, i}(x) b_{n, j}(y) f\left(x_{m, i}, y_{n, j}\right),
\end{aligned}
$$

we can write

$$
\begin{aligned}
\left|\left(L_{m, n}^{\left\langle u_{m}, v_{n}\right\rangle} f\right)(x, y)-f(x, y)\right| \leq & \left|\left(L_{m, n} f\right)(x, y)-f(x)\right| \\
& +\sum_{k=1}^{3}\left|\left({ }_{k} R_{m, n}^{\left\langle u_{m}, v_{n}\right\rangle} f\right)(x, y)\right| .
\end{aligned}
$$

If we show $\lim _{m, n \rightarrow \infty}\left({ }_{k} R_{m, n}^{\left\langle u_{m}, v_{n}\right\rangle} f\right)(x, y)=0, k \in\{1,2,3\}$, then, based on (53), our statement (54) follows, and the proof is ended.

Further, we prove the previous limit only for $k=1$, other two following similar routes.

Since $f \in C_{p, q}\left(\mathbb{R}_{+}^{2}\right)$, a constant $M_{f}$ exists such that

$$
|f(x, y)| \leq M_{f}\left(1+x^{p}\right)\left(1+y^{q}\right), \quad(x, y) \in \mathbb{R}_{+}^{2} .
$$

Based on the classical inequality $(a+b)^{s} \leq 2^{s-1}\left(a^{s}+b^{s}\right)$, $a \geq 0, b \geq 0, s \in \mathbb{N}_{0}$, we deduce

$$
\begin{array}{r}
t^{s} \leq(|t-z|+z)^{s} \leq 2^{s-1}\left(|t-z|^{s}+z^{s}\right) \\
t \geq 0, \quad z \geq 0, \quad s \in \mathbb{N}_{0} .
\end{array}
$$

Consequently, (58) implies that

$$
\begin{aligned}
\left|f\left(x_{m, i}, y_{n, j}\right)\right| \\
\leq M_{f}\left(1+2^{p-1}\left(\left|x_{m, i}-x\right|^{p}+x^{p}\right)\right) \\
\quad \times\left(1+2^{q-1}\left(\left|y_{n, j}-y\right|^{q}+y^{q}\right)\right),
\end{aligned}
$$

where $(i, j) \in \bar{I}\left(x, u_{m}\right) \times \bar{J}\left(y, v_{n}\right)$. 
Using this relation we have

$$
\begin{aligned}
& \left|\left({ }_{1} R_{m, n}^{\left\langle u_{m}, v_{n}\right\rangle} f\right)(x, y)\right| \\
& \leq M_{f} \sum_{i \in \bar{I}\left(x, u_{m}\right)} a_{m, i}(x)\left(1+2^{p-1} x^{p}+2^{p-1}\left|x_{m, i}-x\right|^{p}\right) \\
& \quad \times \sum_{j \in \bar{J}\left(y, v_{n}\right)} b_{n, j}(y)\left(1+2^{q-1} y^{q}+2^{q-1}\left|y_{n, j}-y\right|^{q}\right) \\
& :=M_{f} S_{1} S_{2} .
\end{aligned}
$$

We establish an upper bound for $S_{1}$. Since $i \in \bar{I}\left(x, u_{m}\right)$, clearly

$$
1<u_{m}^{-p}\left|x_{m, i}-x\right|^{p}, \quad p \geq 1,
$$

and we can write

$$
\begin{aligned}
S_{1} \leq & \left(1+2^{p-1} x^{p}\right) \frac{1}{u_{m}^{p}} \sum_{i \in \bar{I}\left(x, u_{m}\right)} a_{m, i}(x)\left|x_{m, i}-x\right|^{p} \\
& +2^{p-1} \sum_{i \in \bar{I}\left(x, u_{m}\right)} a_{m, i}(x)\left|x_{m, i}-x\right|^{p} \\
\leq & \left(\frac{1+2^{p-1} x^{p}}{u_{m}^{p}}+2^{p-1}\right) M_{2 p}^{1 / 2}\left(A_{m} ; x\right) \\
\leq & \left(\frac{1+2^{p-1} x^{p}}{u_{m}^{p}}+2^{p-1}\right) \sqrt{\frac{\Gamma_{2 p}(x)}{m^{p}}}
\end{aligned}
$$

(see (26) and (4)). Arguing similarly we get

$$
S_{2} \leq\left(\frac{1+2^{q-1} y^{q}}{v_{n}^{q}}+2^{q-1}\right) \sqrt{\frac{\Gamma_{2 q}(y)}{n^{q}}} .
$$

Considering (14), relation (61) leads to the claimed result.

\section{Particular Cases}

In presenting these cases, we are looking for one-dimensional linear operators that verify conditions (2) and (4).

(1) Baskakov operators [5] are given by the formula

$$
\begin{gathered}
\left(V_{n} f\right)(x)=\sum_{k=0}^{\infty} b_{n, k}(x) f\left(\frac{k}{n}\right), \\
b_{n, k}(x)=\left(\begin{array}{c}
n+k-1 \\
k
\end{array}\right) x^{k}(1+x)^{-n-k}, \quad x \geq 0,
\end{gathered}
$$

where $V_{n} e_{0}=e_{0}$, hence (2) fulfilled. Since $V_{n}$ reproduces the monomial $e_{1}, e_{1}(t)=t$, its first central moment $\mathscr{M}_{1}\left(V_{n} ; \cdot\right)$ is null.

For $r \geq 2$, the $r$ th central moment is given as follows [ 6 , Lemma 4]:

$$
\mathscr{M}_{r}\left(V_{n} ; x\right)=\sum_{j=1}^{[r / 2]} b_{n, r, j}\left(\frac{x(1+x)}{n}\right)^{j}\left(\frac{1+2 x}{n}\right)^{\delta_{r}},
$$

where $\delta_{r}=1$ if $r$ is odd, $\delta_{r}=0$ if $r$ is even, and $b_{n, r, j}$ are positive coefficients bounded with respect to $n$. In particular, $\mathscr{M}_{r}\left(V_{n} ; x\right)$ is a polynomial of degree $r$ without a constant term. These properties ensure that condition (4) is achieved.

The study of these operators in polynomial weighted spaces was carried out in [6]. Choosing in (10) $A_{m}=V_{m}$ and $B_{n}=V_{n}$ we obtain the Baskakov operator for functions of two variables. The net is $\Delta_{m, n}=(i / m, j / n)_{i, j \geq 0}$. Our results indicated at (47) and (53) are identified with the results established by Gurdek et al. [7, Equations (22), (28)].

The univariate truncated operators has been approached in [8]. The truncated version specified in (16) coincides with the operators studied by Walczak [9, Equation (17)]. In this case $I\left(x, u_{m}\right)$ from (15) becomes $\left\{i \in \mathbb{N}_{0}: i \leq\left[m\left(x+u_{m}\right)\right]\right\}$. Here $[\lambda]$ indicates the largest integer not exceeding $\lambda$.

(2) Szász operators [10] are of the form:

$$
\begin{aligned}
& \left(S_{n} f\right)(x)=\sum_{k=0}^{\infty} p_{n, k}(x) f\left(\frac{k}{n}\right), \\
& p_{n, k}(x)=\frac{e^{-n x}(n x)^{k}}{k !}, \quad x \geq 0 .
\end{aligned}
$$

One has $S_{n} e_{0}=e_{0}$ and $\mathscr{M}_{1}\left(S_{n} ; x\right)=0$. The central moments have the following form:

$$
\begin{gathered}
\mathscr{M}_{2 r}\left(S_{n} ; x\right)=\sum_{j=0}^{r-1} \frac{q_{j}}{n^{r+j}} x^{r-j}, \\
\mathscr{M}_{2 r+1}\left(S_{n} ; x\right)=\sum_{j=0}^{r-1} \frac{p_{j}}{n^{r+j+1}} x^{r-j},
\end{gathered}
$$

where $q_{j}, p_{j}$ are constants; see, for example, [11, Equations (9.5.10)-(9.5.11)]. For this class, conditions (2) and (4) are achieved.

The research of $S_{n}, n \in \mathbb{N}$, operators in polynomial weighted spaces has appeared in [6]. The truncated univariate Szász operators and another extension to functions of two variables in weighted spaces have been considered in [2] and [12], respectively. In the latter paper instead $w_{p, q}$ was used the weight $\rho, \rho(x, y)=1+x^{2}+y^{2}$.

Our theorems of the previous section lead us to twodimensional versions of genuine Szász operators and of their truncated form. In this case the net is $\Delta_{m, n}=(i / m, j / n)_{i, j \geq 0}$.

The next example comes from the world of Quantum Calculus which, in the past two decades, has gained popularity in the construction of linear approximation processes. We choose a $q$-analogue of Szász-Mirakjan operators recently introduced and studied by Mahmudov [13].

(3) $q$-Szász-Mirakjan-Mahmudov operators. Let $q>1$ and $n \in \mathbb{N}$. For $f: \mathbb{R}_{+} \rightarrow \mathbb{R}$ one defines the operator

$$
\left(M_{n, q} f\right)(x)=\sum_{k=0}^{\infty} f\left(\frac{[k]_{q}}{[n]_{q}}\right) \frac{[n]_{q}^{k} x^{k}}{q^{k(k-1) / 2}[k]_{q} !} e_{q}\left(-[n]_{q} q^{-k} x\right) \text {, }
$$


where $e_{q}(z)=\prod_{j=0}^{\infty}\left(1+(q-1)\left(z / q^{j+1}\right)\right)$. We recall the standard notations in $q$-calculus. For $n \in \mathbb{N}$ consider

$$
\begin{gathered}
{[n]_{q}= \begin{cases}\frac{1-q^{n}}{1-q}, & \text { if } q \in(0,1) \cup(1, \infty), \\
n, & \text { if } q=1 ;\end{cases} } \\
{[n]_{q} !=[1]_{q}[2]_{q} \cdots[n]_{q} .}
\end{gathered}
$$

Also, $[0]=0$ and $[0] !=1$. In [13] it was proved that $M_{n, q} e_{0}=e_{0}$ and $M_{n, q}$ is a linear positive operator from $C_{p}\left(\mathbb{R}_{+}\right)$to $C_{p}\left(\mathbb{R}_{+}\right)$for any $p \in \mathbb{N}_{0}$. Withal, for all moments $M_{n, q} e_{r}, e_{r}(t)=t^{r}$, explicit formulas were given as follows:

$$
\left(M_{n, q} e_{r} ; x\right)=\sum_{k=1}^{r} s_{q}(r, k) \frac{x^{k}}{[n]_{q}^{r-j}},
$$

where

$$
\begin{aligned}
& s_{q}(0,0)=1, \quad s_{q}(r, 0)=0 \quad \text { for } r>0, \\
& s_{q}(r, k)=0 \quad \text { for } r<k, \\
& s_{q}(r+1, k)=[k]_{q} s_{q}(r, k)+s_{q}(r, k-1) \\
& \text { for } r \geq 0, \quad k \geq 1,
\end{aligned}
$$

represent $q$-analogue of Stirling numbers; see [13, Lemma 2.6]. One can see that $\left(M_{n, q} e_{r}\right)(x)$ is a polynomial in $x$ of degree $r$ without a constant term, and it is bounded with respect to $[n]_{q}$. These properties are transferred to the central moments $M_{n, q}\left(\varphi_{x}^{r} ; x\right)$, and consequently (4) takes place, provided to replace $n$ with $[n]_{q}$. To construct two-dimensional operators of the form (10) and (16) we choose $A_{m}=M_{m, q}$, $B_{n}=M_{n, q}$, and the network will be

$$
\Delta_{m, n}=\left(\frac{[i]_{q}}{[m]_{q}}, \frac{[j]_{q}}{[n]_{q}}\right)_{i, j \geq 0} .
$$

In time were carried out $q$-analogues of these operators not only for $q>1$ but for the case $q \in(0,1)$; see, for example, $[14,15]$. Extensions of these classes of operators by our method also work there.

\section{References}

[1] J. Gróf, "Über approximation durch polynome mit belegungsfunktion," Acta Mathematica Academiae Scientiarum Hungaricae, vol. 35, no. 1-2, pp. 109-116, 1980.

[2] H. G. Lehnhoff, "On a modified Szasz-Mirakjan-operator," Journal of Approximation Theory, vol. 42, no. 3, pp. 278-282, 1984.

[3] O. Agratini, "On the convergence of a truncated class of operators," Bulletin of the Institute of Mathematics. Academia Sinica, vol. 31, no. 3, pp. 213-223, 2003.

[4] G. A. Anastassiou and S. G. Gal, Approximation Theory. Moduli of Continuity and Global Smoothness Preservation, Birkhäuser, Boston, Mass, USA, 2000.

[5] V. A. Baskakov, "An example of a sequence of linear positive operators in the space of continuous functions," Doklady Akademii Nauk SSSR, vol. 113, pp. 249-251, 1957.
[6] M. Becker, "Global approximation theorems for Szász-Mirakjan and Baskakov operators in polynomial weight spaces," Indiana University Mathematics Journal, vol. 27, no. 1, pp. 127-142, 1978.

[7] M. Gurdek, L. Rempulska, and M. Skorupka, "The Baskakov operators for functions of two variables," Collectanea Mathematica, vol. 50, no. 3, pp. 289-302, 1999.

[8] J. Wang and S. Zhou, "On the convergence of modified Baskakov operators," Bulletin of the Institute of Mathematics. Academia Sinica, vol. 28, no. 2, pp. 117-123, 2000.

[9] Z. Walczak, "Baskakov type operators," The Rocky Mountain Journal of Mathematics, vol. 39, no. 3, pp. 981-993, 2009.

[10] O. Szász, "Generalization of S. Bernstein's polynomials to the infinite interval," Journal of Research of the National Bureau of Standards, vol. 45, pp. 239-245, 1950.

[11] Z. Ditzian and V. Totik, Moduli of Smoothness, vol. 9, Springer, New York, NY, USA, 1987.

[12] N. İspir and C. Atakut, "Approximation by modified SzaszMirakjan operators on weighted spaces," Proceedings of the Indian Academy of Sciences, vol. 112, no. 4, pp. 571-578, 2002.

[13] N. I. Mahmudov, "Approximation by the q-Szász-Mirakjan operators," Abstract and Applied Analysis, vol. 2012, Article ID 754217, 16 pages, 2012.

[14] A. Aral, "A generalization of Szász-Mirakyan operators based on q-integers," Mathematical and Computer Modelling, vol. 47, no. 9-10, pp. 1052-1062, 2008.

[15] A. Aral and V. Gupta, "The q-derivative and applications to qSzász Mirakyan operators," Calcolo, vol. 43, no. 3, pp. 151-170, 2006. 


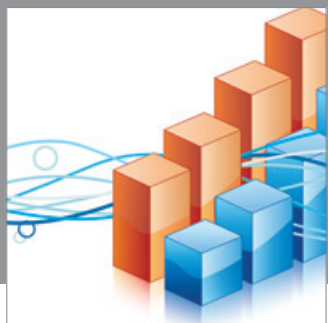

Advances in

Operations Research

mansans

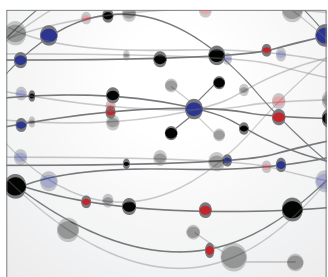

The Scientific World Journal
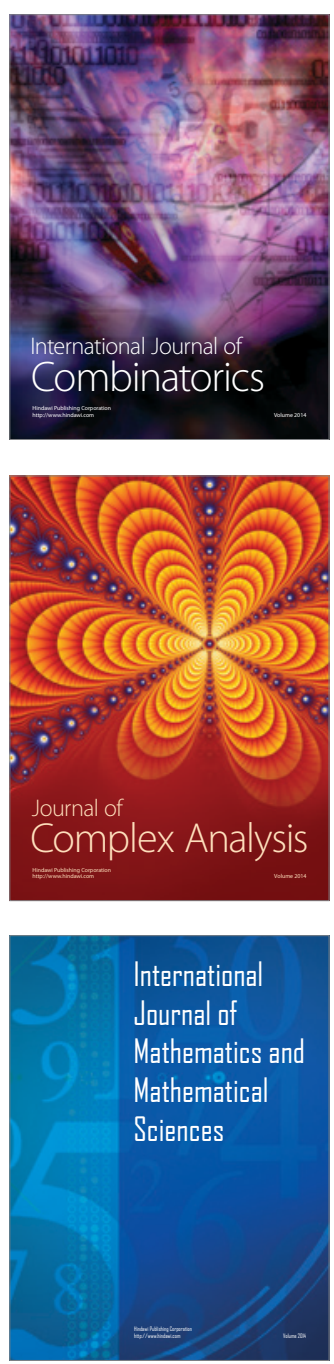
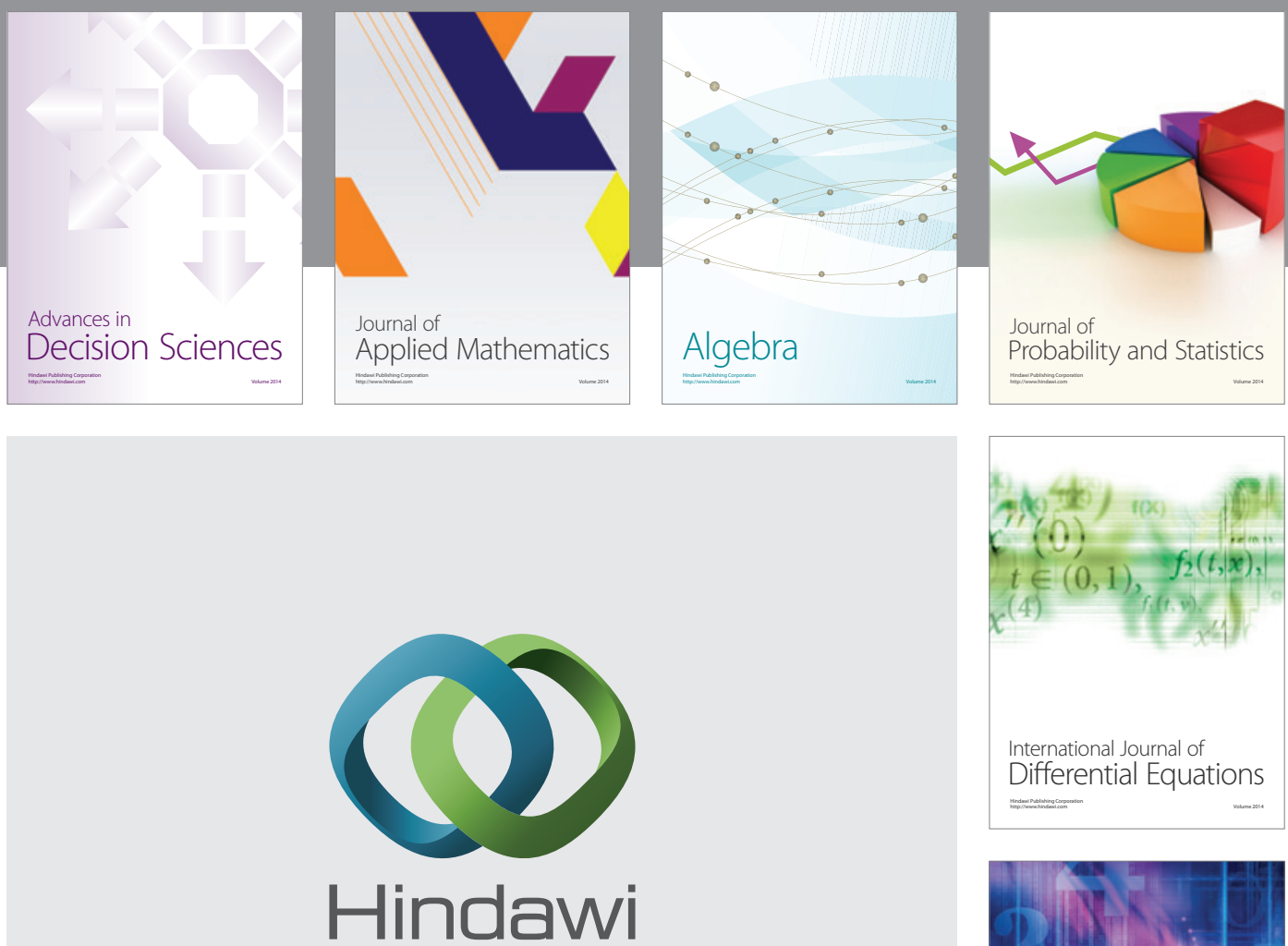

Submit your manuscripts at http://www.hindawi.com
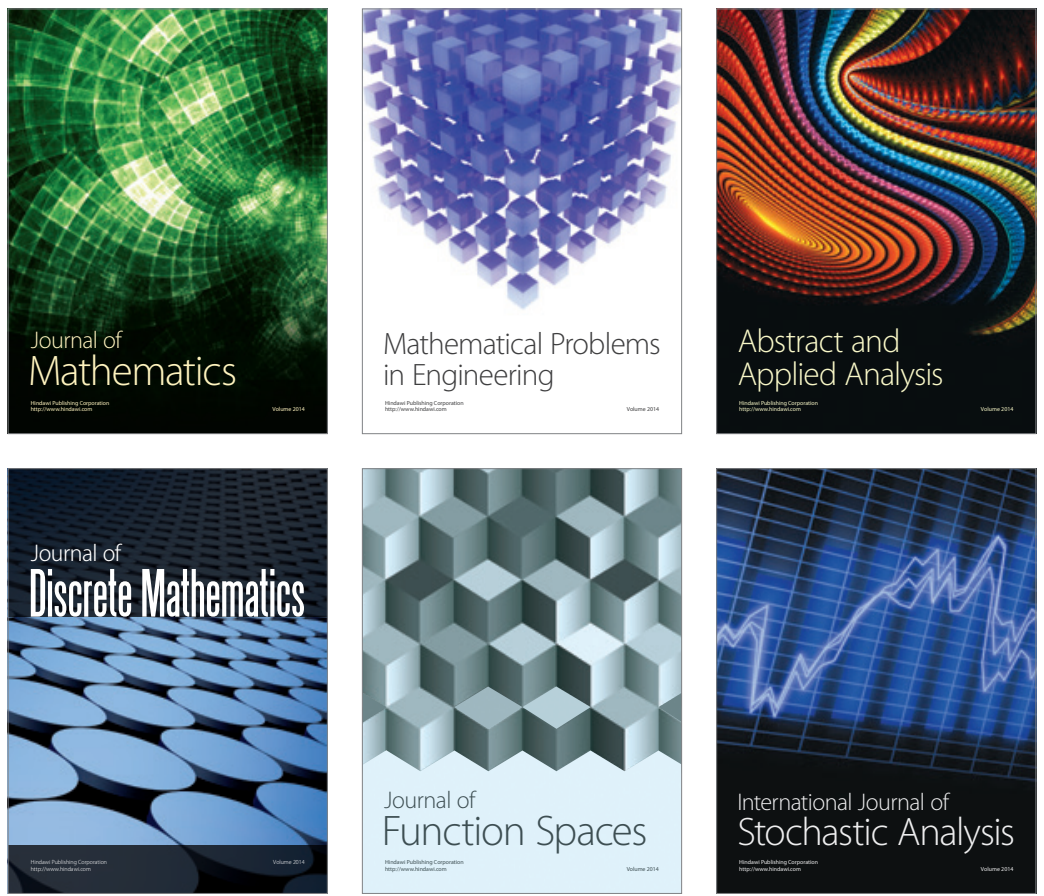

Journal of

Function Spaces

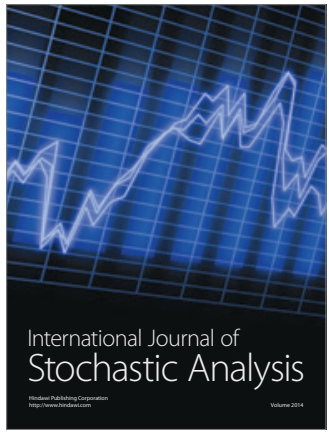

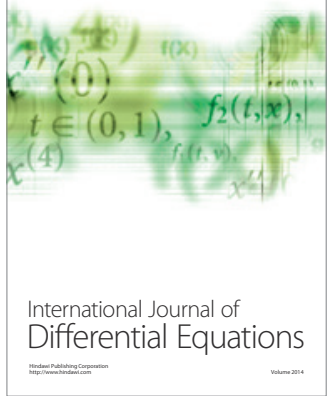
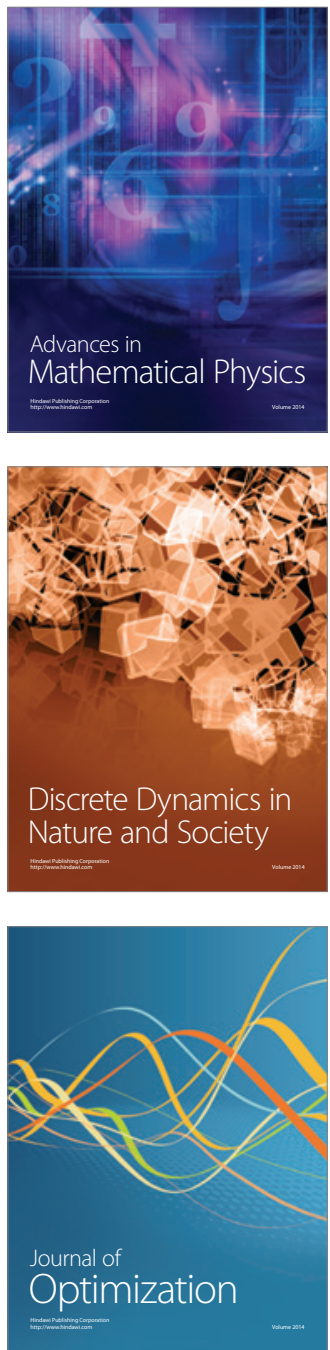